\title{
A journey from brain to muscle across the thyroid continent
}

\author{
Salvatore Benvenga ${ }^{1,2,3}$
}

Published online: 3 March 2017

(C) Springer Science+Business Media New York 2017

It is pertinent to start this Introduction by making reference to the policy of this journal to render articles available online as soon as possible after their final acceptance and proof correction. Indeed, approximately one year ago, I started receiving requests for one article [1] that appeared in the very nice guest issue edited by Professor Evi Diamanti-Kandarakis (Endocrine Disrupting Chemicals-is it time to act?) [2] before I realized that the abstract had already been published in PubMed! I have received further requests for the same article as well as for other articles [3-5] that appear in the present special issue while I had been attending the 86th Annual Meeting of the American Thyroid Association (ATA) in Denver, Colorado, this September. Two of these papers were even mentioned at the discussion of some posters between authors and attendees, and I was invited to provide some further information. No doubt I was pleased by this citation, foremost because it suggested (and still suggests) to me that the articles are of current interest for thyroidologists.

One might ask why this guest issue contains a number of articles that I myself have coauthored [3-8]. Here are the answers. Originally, when I was invited to guest edit a special issue and Professor Christian Koch, the Editor, okayed the

Salvatore Benvenga

s.benvenga@live.it

1 Section of Endocrinology, Department of Clinical and Experimental Medicine, University of Messina, School of Medicine, 98125 Messina, Italy

2 Master Program on Childhood, Adolescent and Women's Endocrine Health, University of Messina, School of Medicine, 98125 Messina, Italy

3 Interdepartmental Program on Molecular \& Clinical Endocrinology, and Women's Endocrine Health, University Hospital, A.O.U.

Policlinico G. Martino, 98125 Messina, Italy topics from a list of themes, I planned to contribute one article. However, I considered a second article, in the event some invited author(s) were about to fail to keep her/his/their promise to contribute a paper or their paper had been rejected. Both types of events did occur during the journey of this guest issue, and it could not be counteracted by additional contacted authors for the corresponding/respective topic(s). Perhaps, in order to have the guest issue ready for publication in December 2016 allowing for revisions and re-revisions, the deadline given to authors was somewhat tight, even though some re-negotiation occurred. There was one reason why I wanted to have the issue published in December 2016. The reason is that 2016 is the anniversary for a number of papers (Table 1) that are given credit in the pertinent articles. As an invited author for the aforementioned guest issue of Prof. Diamanti-Kandarikis $(1,2)$, I had submitted my article well ahead of the deadline. Now, taking into account that I am on the Editorial Board of Reviews in Endocrine \& Metabolic Disorders, I felt an obligation to not leave the journal in a difficult situation. Thus, together with the coauthors, I rushed to finish articles that were intended for other journals and decided submit to REMD upon approval by the Editor-inChief. I do apologize to other Editors who were interested in receiving some of my articles for submission. At this point, it is important to notice that all my manuscripts were handled by the Editor-in-Chief, and underwent regular peer-review, sometimes a tough one. Suffice it to say that one of those articles had a turn-around time of almost 5 months, trying to address the countless comments by the anonymous reviewers. Not to mention the additional comments received by Christian himself! The final result is a guest issue with ten articles that contains 16 Figures (ten colored), 14 Tables, and 941 references. For readers on the run, jumping to some Figures and/or Tables permit catching the core of the article, without reading it all in detail. 


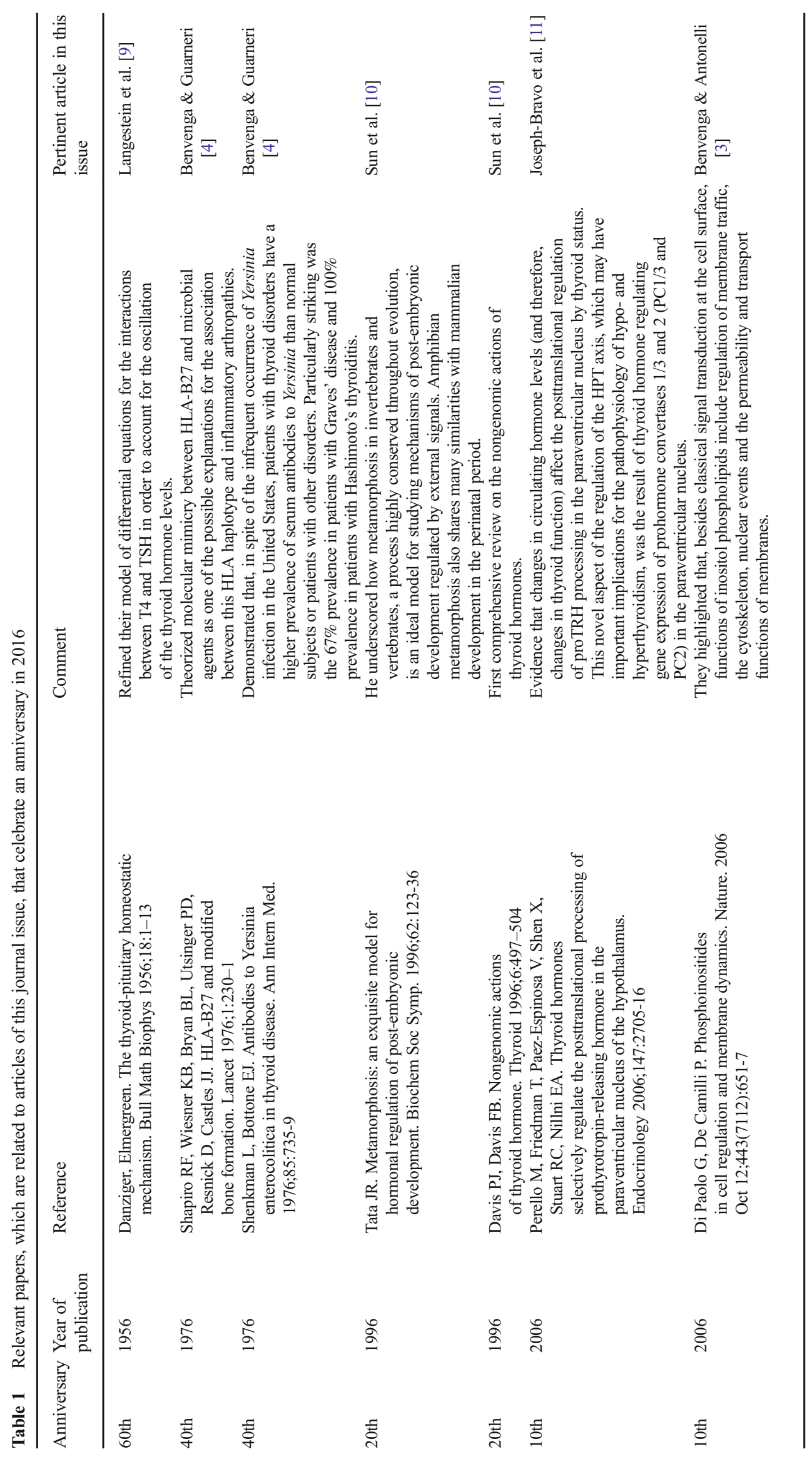


I am referring especially to Fig. 1 (backed by a 1,187-word long legend) of the Joseph-Bravo et al. article [11], and the first two figures (backed by legends for a total of over 520 words) of the Sun et al. article [10]. Noteworthy, $28.5 \%$ of the citations concern papers published in the last 5 years (20122016), with a remarkable $17.7 \%$ pertaining to the last 3 years (2014-2016). Fresh stuff, indeed, and hats off to the authors! From this viewpoint, two articles stand out, namely the article by Ieni et al. [6] and the one by Joseph-Bravo et al. [11]. The former [6] features $86 \%$ of its references published during 2012-2016 and 64\% in 2014-2016, while the corresponding numbers of the latter are and $38 \%$ and $43 \%$ [11]. I wonder whether the journal should change its name from Reviews in Endocrine and Metabolic Disorders to Recent Evidence in Metabolic and endocrine Disorders, a novel denomination that would maintain the acronym REMD.

All that said, let us come to highlight why the articles of this issue are noteworthy. When I read again all contributions in view of writing this Introduction, I realized that, as a reader, I made a journey through the vast Thyroid continent. A multiguided tour, by the way.

The journey begins in the top part of the body, visiting and getting to know new transcription factors engaged in TRH gene expression and signaling pathways that define the dynamics of TRH neurons' response to acute and/or long-term influences [11]. Along the journey, we will see the importance of tanycytes as a central relay for the feedback control of the hypothalamus-pituitary-thyroid (HPT) axis, and HPT responses to alterations in energy balance, and other stimuli. As professional tour guides, Joseph-Bravo et al. [11] are equipped with a powerful torch to let us see the interference of maternal and postnatal stress and nutrition on HPT axis programming. Thus, we can appreciate the susceptibility of the HPT axis to early insults, including the devastating effects of the endocrine disruptors.

The Mexican colleagues relay the torch to Alessandro Antonelli and me, so that we and the readers move downwards until we arrive safely in the thyroid (3). Thus, we can show to the traveling readers the importance of the phosphatidylinositol 3-kinase (PI3K) signaling in transmitting to the thyrocytes the signaling of TSH, the hormone released into the bloodstream under the stimulus of TRH. We highlight several experimental and clinical investigations regarding the involvement of the inositols in physiological and pathological situations (including insulin resistance, diabetes, gynecologic endocrinology and andrology), yet leaving a major focus on thyroid. After the trip, one should agree that inositols have promising therapeutic potential.

A somewhat neglected issue has become the transport of thyroid hormones in the blood, once TSH has completed its duty on the thyrocytes and, among other actions, has stimulated the secretion of T4 and T3. At this point, Alessandro was replaced by Fabrizio [8]. The light coming from the screen of the computer is enough/sufficient to show the traveling readers the result of a computer-assisted bioinformatic search. The result is that all plasma carriers of thyroid hormones share a sequence of amino acids which has been pretty much conserved through evolution. This amino acid motif is larger than previously shown and does not exist in proteins that do not bind thyroid hormones. If the thyroid hormone binding domain has remained conserved, similarly to conservation of the lipid binding domain, it should have some importance. Hints are given for the physiological function that this apolipoprotein binding may serve.

Let us allow some time to permit thyroid hormones to arrive to the skeletal muscles [5] and the intestine (10), and remain in the thyroid to see other things going on, things of autoimmune [3, 7, 9], neoplastic [6] or multifaceted nature [12].

Benvenga and Guarneri [4] start showing the relatively scanty literature on molecular mimicry and endocrinology compared to molecular mimicry and other areas; within endocrinology, the thyroid has been quite neglected. In ad hoc tables, they review and summarize the available literature on the possible role of molecular mimicry as a trigger of autoimmune thyroid disease. Furthermore, they integrate the literature by presenting the results of an in silico search for amino acid sequence homologies between some microbial proteins and thyroid autoantigens, and the potential pathogenetic relevance of such homologies. Relevance stems from the overlap with known autoepitopes and the occurrence of specific HLADR binding motifs.

Now Fabrizio and Alessandro exchange their roles [7]. The article states that the most important candidate viruses for triggering autoimmune thyroid disorders (AITD) are hepatitis $\mathrm{C}$ virus $(\mathrm{HCV})$ and human parvovirus B19 (or Erythrovirus B19 or EVB19). Either virus has been isolated from thyroid tissue, so that consequences are more likely to stem from direct damage rather than molecular mimicry. HCV thyroid infection may upregulate CXCL10 gene expression and secretion in thyroid cells recruiting Th1 lymphocytes, releasing IFN- $\gamma$ and tumor necrosis factor (TNF)- $\alpha$, inducing CXCL10 secretion by thyrocytes, perpetuating the immune cascade. This can cause the onset of AITD in genetically predisposed subjects. Finally, consequences of the infection can be neoplastic in nature, further to autoimmune disease (Hashimoto's thyroiditis and/or Graves' disease [GD]).

German guides now take care of the trip [9]. Mathematical modeling approaches have been applied successfully in similar fields of medical research. Fitting models to individual patient data also allows a fast and effective evaluation of predictive markers. They review empirical and mechanistic models for autoimmune thyroid disease, including those elaborated to calculate the relapse risk in GD patients before antithyroid drug (ATD) treatment. They designed a mathematical model (that is, a differential equation model) integrating 
thyroid hormone parameters, thyroid size, antibody titers and a complex algorithm encompassing genetic predisposition, environmental exposures and current immune activity in order to arrive at a prognostic index for relapse risk after treatment. To do so, they were inspired by the mechanistic model of Pandiyan et al. [13], which allows to simulate the course of TSH over time in patients with Hashimoto's thyroiditis. Their sketch of a GD model under ATD treatment needs to be evaluated in further theoretical studies and clinical trials for its potential to treatment individualization and optimization in GD. Noteworthy, a paper was just published on a mathematical tool that may help physicians to monitor the natural history of Hashitoxicosis [14].

On a small, yet significant scale, I have inquired students of the Postgraduate School of Endocrinology, students of the Doctorate in Endocrinology, and basic and clinical thyroidologists about their knowledge of the "hobnail variant of thyroid neoplasia". Upon their negative response, the question was reformulated as "the hobnail variant of papillary thyroid cancer (PTC)", but results / response rates did not change. Someone wished to doublecheck in the 2009 American Thyroid Association (ATA) guidelines on thyroid nodules and cancer [15], and showed me that no mention at all was made to that variant. Importantly, I inquired before publication of the revised version of the ATA guideline in January 2016 [16]. This was enough to justify having this entity in the proposed list of topics [6]. The colleagues from the Pathology Institute of the University in Messina, with whom I am collaborating, will give some information about such terra incognita. Not surprisingly, this is the article with the fewest papers to be reviewed, a one-digit number. No comment is given on purpose, in order to solicit readers to jump at it.

Joel Ehrenkranz, a true American globetrotter par excellence, and Jane Fualal, an Ugandan surgeon, guide the readers through a number of third world countries, illustrating everyday thyroid problems in these populations and even in wildlife [12]. Concerning animals, do not miss their Figure 1. The approach to the patient with thyroid disease in the developing world must take certain constraints into account such as limited access to sub-specialty expertise and to laboratory and imaging diagnostics. The need for central laboratories and their associated infrastructure (specimen transport, dedicated laboratory equipment, reliable electricity, trained laboratory personnel, and patient follow-up) can be obviated by smartphone/tablet point-of care technology. One example of such mobile health (mhealth) approach is the accurate, affordable and accessible smartphone-based TSH assay.

Circulating thyroid hormones have arrived, at normal or low concentrations, into two target tissues: the skeletal muscles [5] and the intestine [10]. So, it is time to go give a look.

Underestimated is the fact that muscle symptoms may represent the predominant or the sole manifestation of hypothyroidism, raising the issue of a differential diagnosis with other causes of myopathy [5]. Rare muscular manifestations, associated with hypothyroidism, are rhabdomyolysis, acute compartment syndrome, Hoffman's syndrome and Kocher-DebréSémélaigne syndrome. Although the pathogenesis of hypothyroid myopathy is not entirely known, proposed mechanisms include altered glycogenolytic and oxidative metabolism, altered expression of contractile proteins, and neuromediated damage. Careful anamnesis, proper clinical neuromuscular examination and blood tests (CK, TSH, FT3, FT4, $\mathrm{TgAb}, \mathrm{TPOAb}$ ) allow to link the myopathy to hypothyroidism and autoimmune thyroiditis. Only rarely, a muscle biopsy needs to be performed.

Sun et al. [10] start reminding the readeship of the difficulty of studying the role of T3 on intestinal maturation in mammals because of the dependence of the mammalian embryos or even neonates on the maternal supply of nutrients, making it difficult to separate the direct vs. indirect effects of T3 on the embryos or neonates. Interestingly, this postembryonic developmental period in mammals resembles anuran metamorphosis in many aspects. Importantly, amphibian metamorphosis offers a number of advantages to study T3 action in vertebrate development. The analyses of intestinal metamorphosis in Xenopus laevis and Xenopus tropicalis have revealed important mechanistic insights on how T3 induces the formation of adult intestinal stem cells and identified many candidate adult stem cell genes. These studies as well as those in other vertebrates, especially the mouse, have revealed conserved roles and mechanisms in the intestinal development and also implicated a role of $\mathrm{T} 3$ in regulating adult intestinal stem cell functions.

It is now time to thank wholeheartedly the guides for their efforts and patience in receiving memorandum messages about deadlines, the reviewers (including the very thorough ones) for their careful job, and the supporting staff at Springer (particularly, Rosario Gramatica, John Rafael Patricio, Elizabeth Dziubela, and Laura Walsh). We hope that you, the audience and readership, will have as much enjoyment with this guest issue as we did.

\section{Compliance with ethical standards}

Conflict of interest The author declares that he has no conflict of interest related to this work.

\section{References}

1. Benvenga S, Antonelli A, Vita R. Thyroid nodules and thyroid autoimmunity in the context of environmental pollution. Rev Endocr Metab Disord. 2015;16:319-40.

2. Koch CA, Diamanti-Kandarakis E. Introduction to Endocrine Disrupting Chemicals - is it time to act? Rev Endocr Metab Disord. 2015;16(4):269-70. 
3. Benvenga S, Antonelli A. Inositol(s) in thyroid function, growth and autoimmunity. Rev Endocr Metab Disord. 2016. doi:10.1007/ s11154-016-9370-3.

4. Benvenga S, Guarneri F. Molecular mimicry and autoimmune thyroid disease. Rev Endocr Metab Disord. 2016. doi:10.1007/s11154-0169363-2.

5. Sindoni A, Rodolico C, Pappalardo MA, Portaro S, Benvenga S. Hypothyroid myopathy: A peculiar clinical presentation of thyroid failure. Review of the literature. Rev Endocr Metab Disord. 2016. doi:10.1007/s11154-016-9357-0.

6. Ieni A, Barresi V, Cardia R, Licata L, Di Bari F, Benvenga S et al. The micropapillary/hobnail variant of papillary thyroid carcinoma: a review of series described in the literature compared to a series from Southern Italy pathology institution. Rev Endocr Metab Disord. 2016. Nov doi:10.1007/s11154-016-9398-4.

7. Fallahi P, Ferrari SM, Vita R, Benvenga S, Antonelli A. The role of human parvovirus B19 and hepatitis C virus in the development of thyroid disorders. Rev Endocr Metab Disord. 2016. doi:10.1007/ s11154-016-9361-4.

8. Benvenga S, Guarneri F. Conservation in the phylum of the local homology of apolipoproteins with the thyroid hormone plasma carriers. Rev Endocr Metab Disord. 2016. doi:10.1007/s11154-0169379-7.

9. Langenstein C, Schork D, Badenhoop K, Herrmann E. Relapse prediction in Graves' disease: Towards mathematical modeling of clinical, immune and genetic markers. Rev Endocr Metab Disord. 2016. doi:10.1007/s11154-016-9386-8.

10. Sun G, Roediger J, Shi YB. Thyroid hormone regulation of adult intestinal stem cells: Implications on intestinal development and homeostasis. Rev Endocr Metab Disord. 2016. doi:10.1007/ s11154-016-9380-1.

11. Joseph-Bravo P, Jaimes-Hoy L, Charli JL. Advances in TRH signaling. Rev Endocr Metab Disord. 2016. doi:10.1007/s11154-0169375-y.

12. Fualal J, Ehrenkranz J. Access, availability, and infrastructure deficiency: The current management of thyroid disease in the developing world. Rev Endocr Metab Disord. 2016. doi:10.1007/s11154016-9376-x.

13. Pandiyan B, Merrill SJ, Benvenga S. A patient-specific model of the negative-feedback control of the hypothalamus-pituitarythyroid (HPT) axis in autoimmune (Hashimoto's) thyroiditis. Math Med Biol. 2014;31:226-58.

14. Pandiyan B, Merril S, Benvenga S. A homoclinic orbit in a patientspecific model of Hashimoto's thyroiditis. Differ Equ Dyn Syst. 2016. doi:10.1007/s12591-016-0335-5.

15. American Thyroid Association (ATA) Guidelines Taskforce on Thyroid Nodules and Differentiated Thyroid Cancer, Cooper DS,
Doherty GM, Haugen BR, Kloos RT, Lee SL, et al. Revised American Thyroid Association management guidelines for patients with thyroid nodules and differentiated thyroid cancer. Thyroid. 2009;19:1167-214.

16. Haugen BR, Alexander EK, Bible KC, Doherty GM, Mandel SJ, Nikiforov YE, et al. 2015 American Thyroid Association Management Guidelines for Adult Patients with Thyroid Nodules and Differentiated Thyroid Cancer: The American Thyroid Association Guidelines Task Force on Thyroid Nodules and Differentiated Thyroid Cancer. Thyroid. 2016;26:1-133.

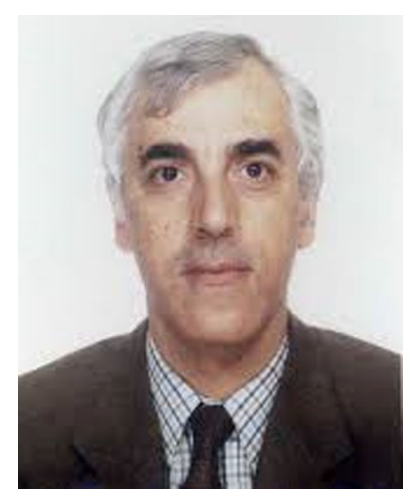

Professor Salvatore Benvenga is Chief of the Interdepartmental Program on Molecular and Clinical Endocrinology and Womens' Endocrine Health at the University of Messina in Sicily. $\mathrm{He}$ also is Director of the Master Program in Childhood, Adolescent and Womens' Endocrine Health. Prof. Benvenga has published more than 245 papers in peer-reviewed journals indexed by PubMed, and 263 papers indexed by Scopus. His H-index is 31 (Scopus) and 37 (Google Scholar), and his citations are more than 3600 (Scopus). Prof. Benvenga is internationally known especially for: 1) the set-up of the first assay for serum thyroid hormone autoantibodies (THAb) based on a specific radioimmunoprecipation method: 2 ) the set-up of the first radioimmunoassay for human prealbumin; 3) discovery and characterization of thyroid hormone binding to apolipoproteins; 4) having emphasized the importance of endocrine complications (anterior hypopituitarism) following head trauma; 5) having provided evidence in favor of autoimmune thyroid disease being triggered by microorganisms via molecular mimicry in genetically predisposed persons; 6) having demonstrated the superiority of modern formulations of L-thyroxine (compared to the classical tablet formulation) in a variety of clinical settings. Among the numerous awards and prizes he has won, Prof. Benvenga received the Giuseppe Zagari Prize for Medicine in 2012. He serves on the Editorial Board of several prestigious journals and is an international lecturer. Besides mentoring and teaching numerous nurses, medical students, residents, fellows, postdocs, and junior faculty members, Prof. Benvenga also served as a co-tutor for a Doctorate in Mathematics at the Marquette University, Milwaukee, WI, USA. 